\title{
A Study on Objective Evaluation of Vehicle Steering Comfort Based on Driver's Electromyogram and Movement Trajectory
}

\author{
Yahui Liu, Member, IEEE, Qi Liu, Chen Lv, Minghui Zheng, and Xuewu Ji
}

\begin{abstract}
The evaluation of driver steering comfort, which is mainly about the haptic driver-vehicle interaction, is important for the optimization of advanced driver assistance systems (ADAS). The current approaches to investigating steering comfort are mainly based on the driver's subjective evaluation, which is time-consuming, expensive, and easily influenced by individual variations. This paper makes some tentative investigation of objective evaluation, which is based on the electromyogram (EMG) and movement trajectory of the driver's upper limbs during steering maneuver. First, a steering experiment with 21 subjects is conducted, and EMG and movement trajectories of the driver's upper limbs are measured, together with their subjective evaluation of steering comfort. Second, five evaluation indices including EMG and movement information are established based on the measurements from the first step. Correlation analyses are conducted between each evaluation index and steering comfort rating (SCR), and the results show that all of the indices have significant correlations with SCR. Then, an artificial neural network (ANN) model is established based on the aforementioned indices and its predicting performance of SCR is demonstrated as acceptable. The results reveal that it may be feasible to establish an objective evaluation approach for vehicle steering comfort.
\end{abstract}

Index Terms-EMG, haptic driver-vehicle interaction, movement trajectory, objective evaluation, steering comfort.

\section{INTRODUCTION}

$\mathrm{A}$ DVANCED driver assistance systems (ADAS) have attracted continual research attention in the field of automotive industry since 1990s [1]-[3]. Haptic shared control is a powerful technique that well combines the driver's maneuver and the ADAS [4], while keeping the driver in the loop and avoiding complicated automation issues [5-7]. To achieve this, the mechanism of haptic driver-vehicle interaction

Manuscript received xxx xx, 2016; revised xxx xx, 2016. This work was supported by the National Natural Science Foundation of China under Grant 51375009 and Tsinghua University Initiative Scientific Research Program under Grant 2014z21039. (Corresponding author: Xиеwu Ji).

Y. Liu, Q. Liu, and X. Ji are with the State Key Laboratory of Automotive Safety and Energy, Tsinghua University, Beijing 100084, China, and also with the Department of Automotive Engineering, Tsinghua University, China. (e-ma il: liuyahui@ tsinghua.edu.cn; q-liu16@ mails.tsinghua.edu.cn; jixw@tsinghua. edu.cn).

C. Lv is with the Advanced Vehicle Engineering Center, Cranfield Universi ty, UK (henrylvchen@163.com).

M. Zheng is with the Department of Mechanical Engineering at University of California, Berkeley, USA (zhengmhbuaa@gmail.com). should be taken into account [8]-[11], and workable approaches for studying the driver's neuromuscular characteristics in various operational conditions are necessary [12]. Steering is one of the most important operations for the driver to control the vehicle [13]-[14], and the steering feeling which consists mainly of haptic driver-vehicle interaction has greatly influence in reducing the driver's physiological and psychological burden. Therefore, steering comfort, in particular the driver's steering feeling and the dynamics property of the driver's body in steering maneuver, should be investigated for the optimization of ADAS.

Although the steering comfort evaluation is an important way to identify the neuromuscular characteristics of the driver, our knowledge is still mainly based on the driver's subjective perceptions, which is time-consuming, expensive, and exhibit large individual variations for different drivers [15]. Therefore, it is important to establish an objective method of evaluating the steering comfort, which includes both posture comfort and operation comfort. Posture comfort is related to static driving postures and interior layout of vehicle, while the operation comfort is determined by both driving postures and dynamic characteristics of steering system.

The current studies of posture comfort mainly focus on the angle ranges of body joints for comfortable driving posture. Porter and Gyi [16] conducted an experiment to investigate the optimal driving postures, and found that the derived joint angles may not be applicable to all drivers. Park et al. [17] analyzed the anthropometric data of Korean drivers and investigated the relationship between body segment lengths, preferred postural angles and adjustment level of the driver's seat. Mohamad et al. [18]-[19] conducted experiments with a large number of subjects to investigate the ranges of comfortable angles for Malaysian citizens, and proposed a set of comfortable dimensions for the driver's seat. The studies above show that there exist significant preference variations for drivers from different countries and regions, indicating that a particular comfortable set of angle ranges are only valid for a particular group.

These studies on comfortable joint angles or preferred postural angles improved the ergonomic design of vehicle driver workspace, and provided an approach to evaluating driving postures. The angle ranges were mainly defined by subjective feelings and preferences of drivers [20], and the preferred posture was only a part of the influence factors for 
comfort or discomfort in the driver's feelings [21]. Therefore, additional biomechanical methods need to be developed for the optimization of vehicle ergonomic design.

Kolich [22] proposed a prediction method for automobile seat comfort using an artificial neural network (ANN) model, and found that ANN model had superior predicting performance compared with a linear regression model [15]. Kyung and Nussbaum [23] evaluated the driver workspace with digital human models (DHM) based on an enlarged group of body joint angles, and specified the comfortable driving postures with accurate ranges of joint angles. They also investigated the driver-seat pressure distribution, and identified some pressure variables which were derived from the average (peak) contact pressure ratio. These pressure variables could be used for seat comfort assessment [24]. Body pressure distribution was further investigated by Park et al. [25], with the help of 3D scanning measuring technique. Alessandro and Sandro [26] developed an evaluating model, which could provide a numerical evaluation for the discomfort level of the driver. The model was based on human manikin and discomfort functions, which were derived from LUBA (postural loading on the upper body assessment) and geometric-spatial evaluations.

Studies on the driving posture comfort provide many ways to facilitate the ergonomic design of driver's workplace. The driver's preferred postures are usually obtained in static situations without considering the dynamic operations in actual driving tasks. Yang et al. [27] used motion capture system to measure the movement trajectories of the driver's upper limbs under different driving postures and steering torques. They used twelve dynamic discomfort indices based on movement measurements, and conducted correlation analyses between each index and the driver's subjective rating of discomfort. After that, they established an integrated discomfort index using a linear regression method. Liu et al. [28] identified the functions of ten muscles around the shoulder in dynamic steering maneuvers based on the analyses of surface electromyogram (EMG) signals, and developed a novel estimation method for driver steering efficiency [29]. The steering efficiency reveals the driver's energetics and control strategies, both of which are applicable to steering comfort evaluation [30]. The EMG amplitudes were also used in a long-time driving task to find the relationship between muscle activities and driver comfort [31].

For the level of comfort in dynamic steering maneuver, there are very few established objective evaluation approaches in the literature, particularly those for the comfort of short-time steering operation. The purpose of this study is to investigate the relationship between the driver's physiological characteristics and his subjective feelings of steering, and to discuss the feasibility of establishing an objective evaluation method of steering comfort.

The EMG and the movement trajectory can well reflect the driver's physiological characteristics. The EMG reflects the driver's neuromuscular characteristics and his muscle workload in a nonintrusive way [32], whereas the movement trajectory signals can be used to analyze the kinematic characteristics of steering maneuver, and provide an assessment of the quality of the steering movement [27].

The most commonly used objective evaluation is to predict subjective comfort perceptions using quantitative measures [22], such as interface pressure of the seat. Several predicting methods of subjective perceptions are available, including statistic models and machine learning. Multiple linear regression is a typical statistic model, but it cannot provide accurate prediction unless there exists significant linear relationship between independent variables and dependent variables. Machine learning methods, such as ANN, are particularly suited to solving both linear and nonlinear problems, and machine learning methods have found wide range of applications in industry [33], such as the identification of drivers' intention [34-35].

In this paper, the objective evaluation of steering comfort is studied. In Section II, a steering experiment is introduced. In Section III, five quantitative evaluation indices of steering comfort are established based on EMG and movement trajectories. The relationship between these indices and driver's subjective steering comfort rating (SCR) is investigated in Section IV. In Section V, an ANN model is built based on quantitative indices, in order to discuss the feasibility of establishing an objective evaluation method of the steering comfort. Conclusions are given in Section VI.

\section{STEERING EXPERIMENT}

In this paper, the driver's neuromuscular and movement characteristics are employed to replace his subjective feelings of steering comfort. Such characteristics are measured by EMG as well as movement trajectories, and can be described by quantitative indices. Once the relationship between subjective feeling and quantitative indices is clear, an objective evaluation approach might be established. A steering experiment was conducted in dynamic conditions, where the driver performed steering tasks with both hands in simulated driving scenarios. The experiment is aiming to investigate the aforementioned relationship, and to discuss the feasibility of the objective evaluation.

\section{A. Experimental Equipment}

The experiment was conducted on a six degrees-of-freedom driving simulator, which provides virtual dynamic driving scenarios by CarSim (Fig. 1). A hydraulic servo-system was equipped under the cockpit to simulate the vehicle dynamic responses. A force sensor (DynPick WEF-6A1000) and a torque-angle sensor (TR-60TC) were mounted under the steering wheel. The inclination angle of the steering axis was set to 30 degree. The EMG were measured by Nihon Kohden $Z B-150 H$ wireless sensors with a sampling frequency of 1000 $\mathrm{Hz}$. The EMG, together with steering angle and steering torque, were recorded by Web-1000 multi-channel system. The movement trajectories of driver's upper limbs were detected by Osprey-Motion Analysis system with a sampling frequency of $100 \mathrm{~Hz}$. 
DRIVER'S ELECTROMYOGRAM AND MOVEMENT TRAJECTORY

\section{B. Experimental Design}

In the experiment, 21 male voluntary subjects were recruited, and none of them had musculoskeletal anomalies and joint diseases. The subjects gave their informed consent to participation and the procedure was approved by the local ethical committee. The subjects were aged from 20 to 40 , including seven skilled drivers, seven drivers with average driving experience and seven unskilled drivers.

As shown in Fig. 2 and Fig. 3, the EMG were measured from ten muscles (five muscles from each upper limb), including the anterior deltoid (DELT-A), the posterior deltoid (DELT-P), the clavicular portion of pectoralis (PMA-C), the teres major (TM), and the long head of triceps brachii (TB-L). As shown in Table I, all of these muscles have been verified to play important roles in the steering task [28]. The electrodes were placed in the center of relevant muscles, and were kept as far apart as possible from the nearby muscles to prevent interference.

The movement trajectories of driver's upper limbs were detected during steering maneuvers by cameras. As shown in Fig. 3, the reflective markers were attached to subjects' shoulders, elbows, and wrists.

As shown in Fig. 4, the resisting steering torque was set into five patterns to provide different steering feelings, and to simulate the various steering assistant at different velocities (for the vehicle's velocity may have influence on the steering maneuvers). In each steering pattern, the subject was asked to perform a slalom driving task (Fig. 5).

After each task, the subject was asked to give a SCR, which is scaled from 1 to 10 (where 1 represents the worst steering comfort feeling, and 10 the best). After completion of the five tasks, the subject repeated the task in Pattern $\mathrm{C}$ and gave the SCR again. This is used to verify the consistency of his subjective evaluation by comparing the two SCRs under Pattern C. If the difference is significant, the experiment should be conducted again.

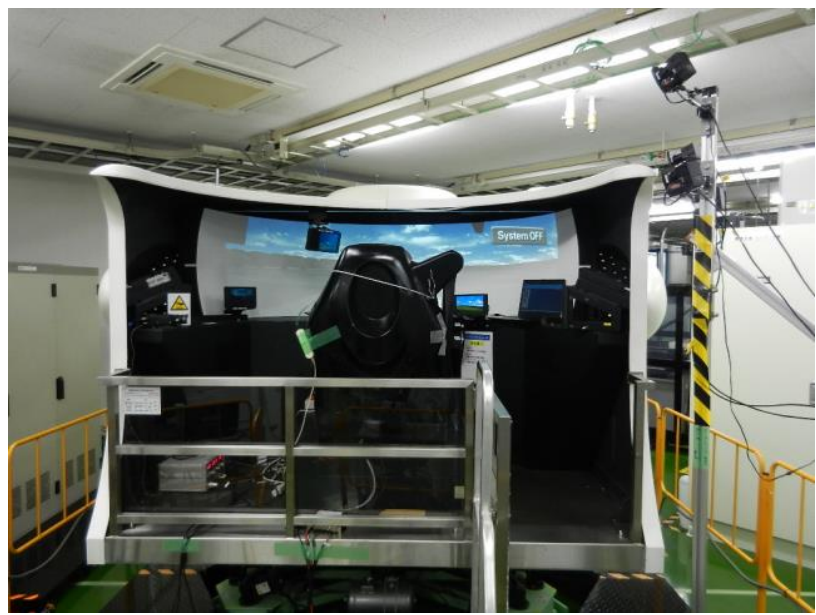

Fig. 1 Driving Simulator.

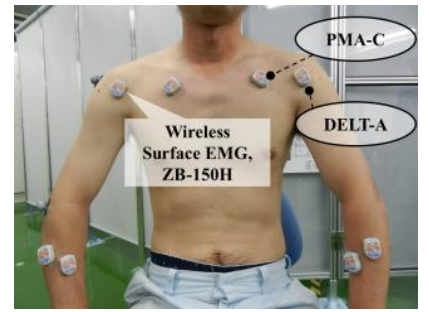

Fig. 2 The placement of EMG sensors and motion reflective markers (front view).

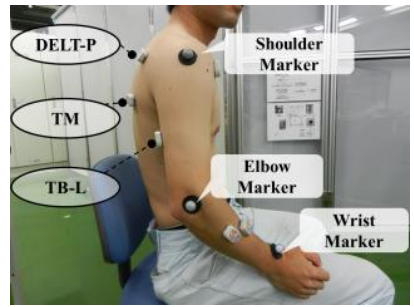

Fig. 3 The placement of EMG sensors and motion reflective markers (side view).

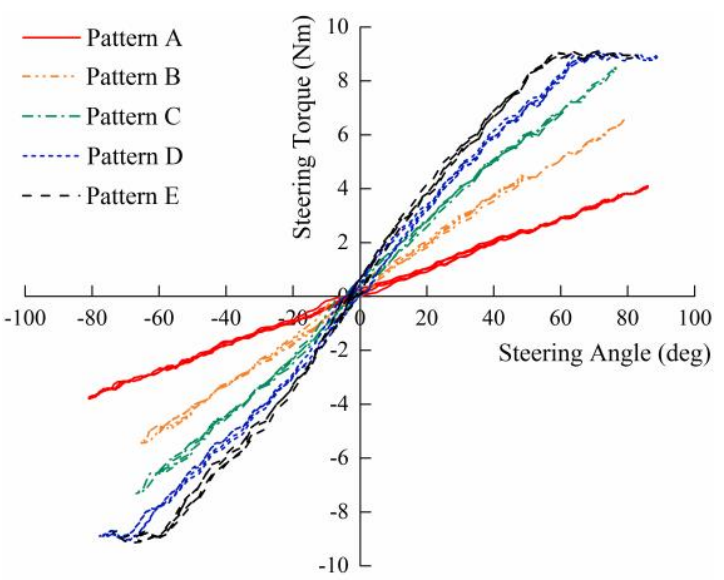

Fig. 4 The five steering patterns with different resisting steering torque.

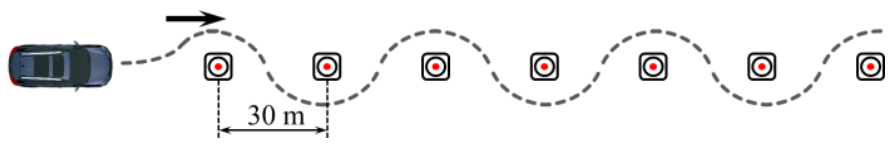

Fig. 5 Slalom driving task.

TABLE I

FUNCTION OF MEASURED MUSCLES DURING STEERING MANEUVER

\begin{tabular}{lll}
\hline \hline & Steering direction & \\
\cline { 2 - 3 } & Clockwise & Counter-clockwise \\
\hline Prime mover (s) & PMA-C & TB-L \\
& DELT-A & \\
& DELT-P & \\
Fixator & TM & TM \\
\hline \hline
\end{tabular}

\section{EVALUATION INDICES}

Based on the measured EMG and movement trajectories, five quantitative evaluation indices were established, including two EMG indices and three movement indices. These indices reflect the driver's neuromuscular and movement characteristics, and they are potentially useful in building an objective evaluation of driver's steering comfort.

\section{A. EMG evaluation indices}

Muscle tissues generate force only on contraction modes. Therefore, an agonist-antagonist muscle pair, in which the two muscles are positioned opposite to each other, is required to produce both positive and negative moments around the relevant joint. The opposition between muscles is generally named as muscle co-contraction. 
It has been identified that the co-contraction is employed by the driver during steering maneuver as part of an optimum muscle activation strategy, which increases the stiffness of the limb and the bandwidth of the steering control loop [36]. However, muscle co-contractions sometimes cannot be compared in different steering conditions, even for the same driver. Hence, in some cases the steering efficiency [29]-[30] might be more appropriate for the evaluation of steering comfort. In this study, both the average steering efficiency and the average muscle co-contraction are adopted as the EMG indices. The calculation method of these two indices is based on a linear regression model, which is described below.

First, the raw EMG was high-pass filtered at $20 \mathrm{~Hz}$ to remove any interference as well as the component of heartbeat. The root mean square value of EMG in each $0.01 \mathrm{~s}$ segment, defined as $R E M G$, was calculated and then low-pass filtered at $5 \mathrm{~Hz}$ to generate a smooth $R E M G$ (define as $s R E M G$ ). Both high-pass and low-pass filtering were conducted in forward and reverse direction to avoid any phase change [32].

\section{1) Calculation of the tangential hand force}

As shown in Fig. 6, the hand force generated in the wheel plane can be divided into the radial force and the tangential force during steering maneuvers. Only tangential force can provide steering torque, so the calculation of tangential hand force is conducted:

$$
\begin{gathered}
F_{y l}=\frac{1}{2}\left(F_{y}+\frac{M_{z}}{r}\right) \\
F_{y r}=\frac{1}{2}\left(F_{y}-\frac{M_{z}}{r}\right)
\end{gathered}
$$

where $F_{y l}$ and $F_{y r}$ are the tangential force of left hand and right hand, respectively, $F_{y}$ is the sum of the tangential forces of the two hands, $M_{z}$ is the steering torque, and $r$ is the radius of the steering wheel. Both $F_{y}$ and $M_{z}$ can be measured by sensors.

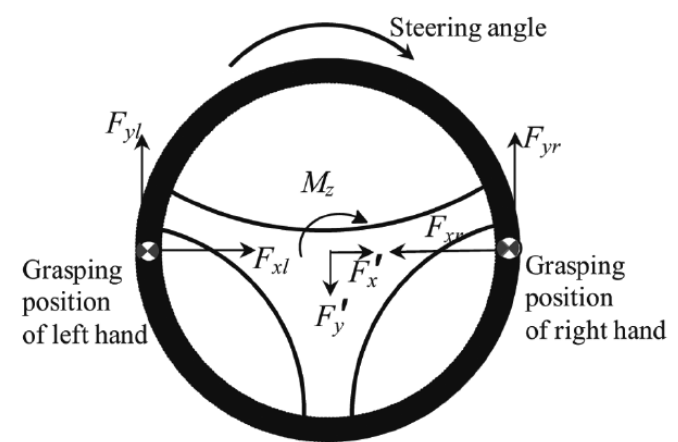

Fig. 6 Force and torque in the plane of steering wheel.

Together with the steering angle, a calculation example of the tangential hand force of the left hand is given in Fig. 7. The $s R E M G$ of the left upper limb are also given in Fig. 7.
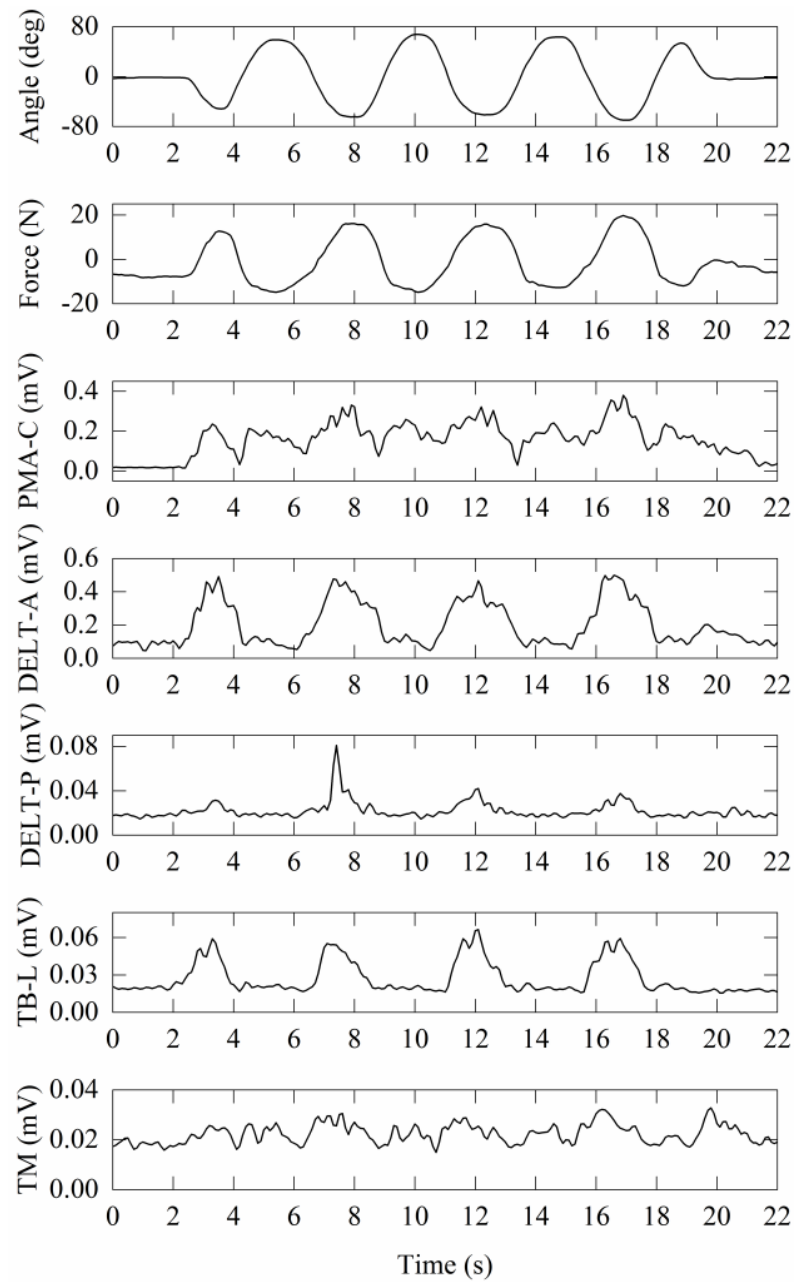

Fig. 7 The calculated tangential hand force of the left hand, and the $s R E M G$ of the left upper limb.

\section{2) Regression of the hand force by EMG}

It is widely acknowledged that there is a linear relationship between muscle force and $s R E M G$ in isometric conditions (where the muscle length remains constant), and the relationship is still obvious in dynamic conditions [32]. In particular, the linear regression result of $s R E M G$ is proportional to tangential force generated on steering wheel by driver's hand [30]. Therefore, a multiple linear regression model is used to estimate tangential hand force based on $s R E M G$ voltage of measured muscles, which is expressed as:

$$
\begin{gathered}
\hat{F}_{y l}=\sum_{i=1}^{n} a_{l i} \times s R E M G_{l i} \\
\hat{F}_{y r}=\sum_{i=1}^{n} a_{r i} \times s R E M G_{r i}
\end{gathered}
$$

where $\hat{F}_{y l}$ and $\hat{F}_{y r}$ are the estimated tangential force of left hand and right hand by multiple regression; $s R E M G_{l i}$ and $S R E M G_{r i}$ are the $s R E M G$ of number $i$ muscle, which is measured during the steering maneuver in left and right; $a_{l i}$ and $a_{r i}$ are the regression coefficients; $n$ is the number of 
measured muscles in each upper limb, and here $n$ is five.

Then the total force capacity can be expressed as:

$$
\begin{aligned}
& F_{l t}=\sum_{i=1}^{n}\left|a_{l i}\right| \times s R E M G_{l i} \\
& F_{r t}=\sum_{i=1}^{n}\left|a_{r i}\right| \times s R E M G_{r i}
\end{aligned}
$$

where $F_{l t}$ and $F_{r t}$ are the total force capacity of left hand and right hand, respectively. The total force capacity is an absolute value without direction, and it represents the total torque produced by measured muscles in steering experiment, including both effective torque component and resisting torque component.

\section{3) Average steering efficiency}

With the aforementioned estimated tangential force and total force capacity, the instantaneous steering efficiency $\eta_{\text {ins }}(t)$ and the average steering efficiency $\eta$ can be calculated, by:

$$
\begin{gathered}
\eta_{i n s}(t)=\frac{\left|\hat{F}_{y l}(t)-\hat{F}_{y r}(t)\right|}{F_{l t}(t)+F_{r t}(t)} \times 100 \% \\
\eta=\frac{\int_{0}^{T}\left|\hat{F}_{y l}(t)-\hat{F}_{y r}(t)\right| d t}{\int_{0}^{T}\left[F_{l t}(t)+F_{r t}(t)\right] d t} \times 100 \%
\end{gathered}
$$

where $t$ is the sampling time of EMG, and $T$ is the duration of steering maneuver. For more detailed definition and calculation of steering efficiency, see reference [29] and [30].

\section{4) Average muscle co-contraction}

The instantaneous muscle co-contraction $I_{\text {Cins }}$ and the average muscle co-contraction $I_{C}$ can be calculated by:

$$
\begin{gathered}
I_{\text {Cins }}(t)=F_{l t}(t)-\left|\hat{F}_{y l}(t)\right|+F_{r t}(t)-\left|\hat{F}_{y r}(t)\right| . \\
I_{C}=\frac{1}{T} \int_{0}^{T}\left[F_{l t}(t)-\left|\hat{F}_{y l}(t)\right|+F_{r t}(t)-\left|\hat{F}_{y r}(t)\right|\right] d t .
\end{gathered}
$$

For more detailed information of the muscle co-contraction, see reference [32]. An estimation example of steering force, as well as instantaneous trend of steering efficiency and muscle co-contraction is shown in Fig. 8. It can be seen that EMG-based estimation gives a good fitting to the measured tangential force.
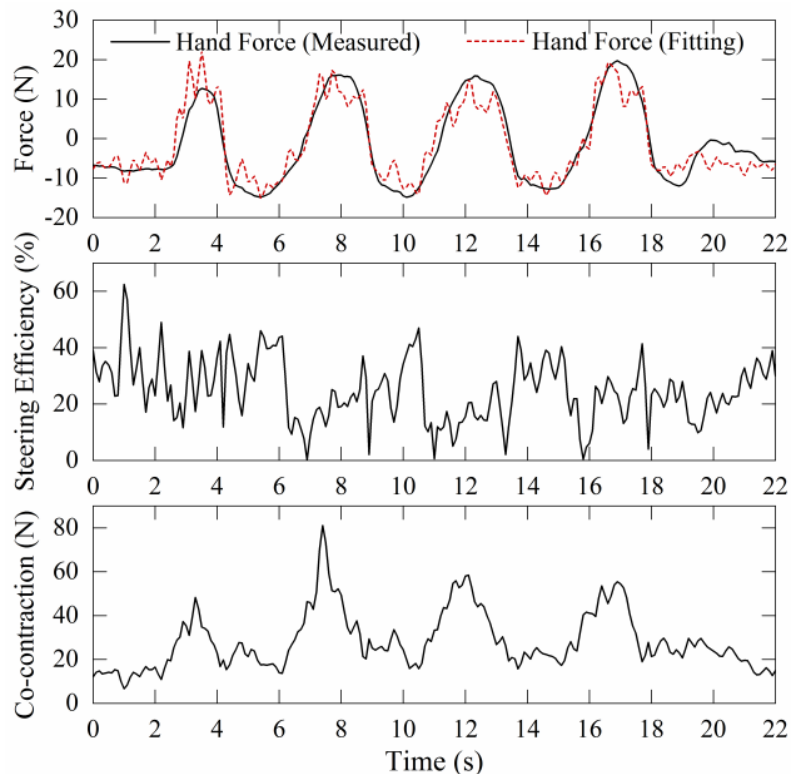

Fig. 8 The estimation of the tangential hand force, as well as the instantaneous trend of the steering efficiency and the muscle co-contraction.

\section{B. Movement evaluation indices}

The movement evaluation indices for steering comfort are derived from the movement trajectories, which were described by the coordinates of joints during the steering tasks. The raw signals were low-pass filtered at $3 \mathrm{~Hz}$ to remove the anomalous coordinate points.

During the steering task, it has been found that the trajectories from wrist or shoulder are not applicable to calculate the movement indices. In fact, the wrist joint was almost fixed with the steering wheel and its moving trajectories remained almost the same for the same driving maneuver; and on the other hand, the movement of shoulder was almost negligible since the driver was asked to keep his torso motionless. However, the elbow joint trajectories showed significant differences as the steering condition changed [27], indicating that elbow had greater flexibility in steering tasks. Therefore, in this study the movement evaluation indices were calculated from the movement signals of the elbow joint.

Totally three movement indices were adopted, including the average velocity $v$, the average acceleration $a$, and the average jerk $j$. In fact, the energy indices (such as the average kinetic energy) may be also relevant to the steering comfort, however, the mass distribution of the upper limb has significant individual variations among different subjects, and the measurement of such mass distribution is rather challenging. Therefore, the energy indices are not adopted here.

\section{1) Average velocity}

The average velocity of the subject's wrist is a first order index of the movement for steering maneuver. The instantaneous velocity $v_{x}(k), v_{y}(k), v_{z}(k)$ in the directions of $X, Y$, $Z$ axis are calculated by: 


$$
\begin{aligned}
& v_{x}(k)=\frac{1}{\Delta k}[x(k+1)-x(k)], \\
& v_{y}(k)=\frac{1}{\Delta k}[y(k+1)-y(k)], \\
& v_{z}(k)=\frac{1}{\Delta k}[z(k+1)-z(k)]
\end{aligned}
$$

where $k$ is the sampling time of movement trajectory, and $\Delta k$ is the sampling interval, and $x, y, z$ are the $X, Y, Z$ coordinated of the marker attached on the elbow joint.

The average velocity $v$ is calculated by:

$$
v=\frac{1}{N} \sum_{k=1}^{N-1}\left\{\left[v_{x}(k)\right]^{2}+\left[v_{y}(k)\right]^{2}+\left[v_{z}(k)\right]^{2}\right\}^{0.5}
$$

where $N$ is the sample length.

\section{2) Average acceleration}

The average acceleration of the wrist is a second order index of the steering movement. The instantaneous acceleration $a_{x}(k)$, $a_{y}(k), a_{z}(k)$ are calculated by:

$$
\begin{aligned}
& a_{x}(k)=\frac{1}{\Delta k}\left[v_{x}(k+1)-v_{x}(k)\right], \\
& a_{y}(k)=\frac{1}{\Delta k}\left[v_{y}(k+1)-v_{y}(k)\right], \\
& a_{z}(k)=\frac{1}{\Delta k}\left[v_{z}(k+1)-v_{z}(k)\right] .
\end{aligned}
$$

The average acceleration $a$ is calculated by:

$$
a=\frac{1}{N} \sum_{k=1}^{N-1}\left\{\left[a_{x}(k)\right]^{2}+\left[a_{y}(k)\right]^{2}+\left[a_{z}(k)\right]^{2}\right\}^{0.5} .
$$

\section{3) Average jerk}

The average jerk of the wrist is a third order index of the steering movement, which reflects driver's movement smoothness, as well as his ride comfort. The instantaneous jerk $j_{x}(k), j_{y}(k), j_{z}(k)$ are calculated by:

$$
\begin{aligned}
& j_{x}(k)=\frac{1}{\Delta k}\left[a_{x}(k+1)-a_{x}(k)\right], \\
& j_{y}(k)=\frac{1}{\Delta k}\left[a_{y}(k+1)-a_{y}(k)\right], \\
& j_{z}(k)=\frac{1}{\Delta k}\left[a_{z}(k+1)-a_{z}(k)\right] .
\end{aligned}
$$

The average jerk $j$ is calculated by:

$$
j=\frac{1}{N} \sum_{k=1}^{N-1}\left\{\left[j_{x}(k)\right]^{2}+\left[j_{y}(k)\right]^{2}+\left[j_{z}(k)\right]^{2}\right\}^{0.5} .
$$

A calculation example of the movement evaluation indices is given in Fig. 9.
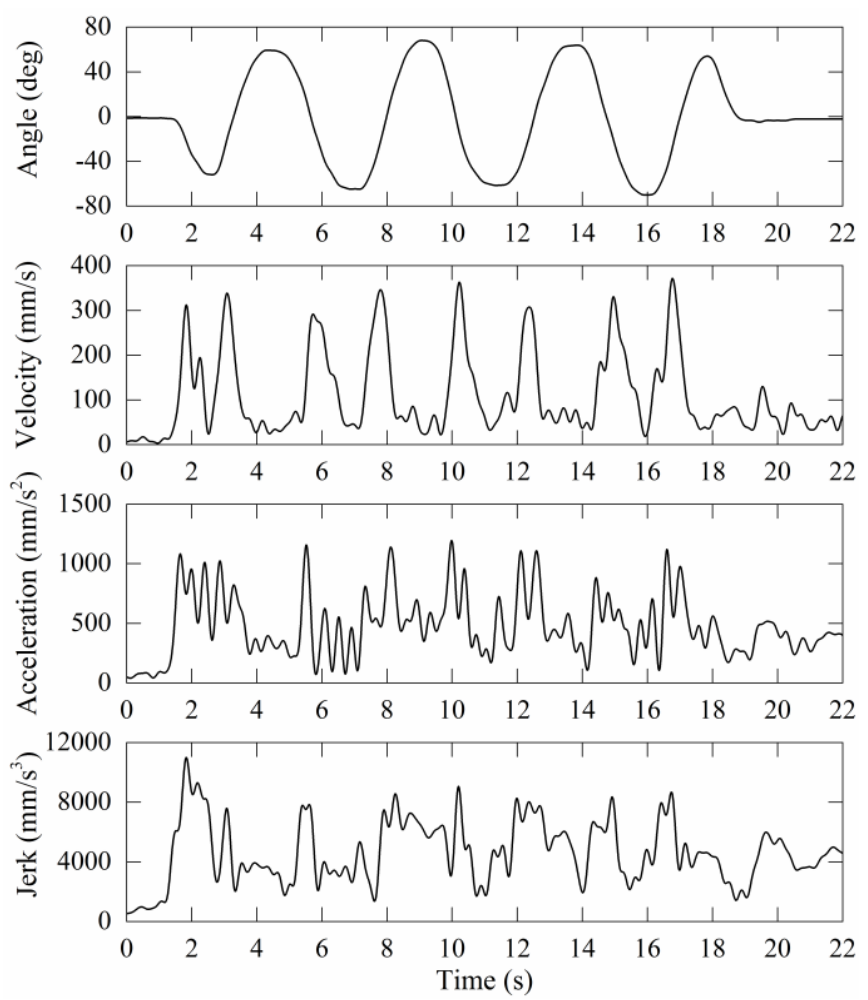

Fig. 9 The calculation of the movement indices.

\section{Correlation Analyses}

The relationship between SCR and quantitative evaluation indices is given in this section. Totally 126 valid samples were collected from 21 subjects. The mean values and the standard deviations of the five indices are calculated and the results are given in Fig. 10. The significance of the correlation is tested by $t$-test, in which the observed $t$-statistic is defined as:

$$
t=\frac{|R| \sqrt{n-2}}{\sqrt{1-R^{2}}}
$$

where $n$ is the sample number, and $R$ is the correlation coefficient. The $t$-statistic is used to test the null hypothesis $\left(\mathrm{H}_{0}\right)$, which represents the situation where there is no trend in the testing data and the slope of the regression formula is zero. For a particular given level of significance (usually set to 0.05), the null hypothesis can be rejected if the $t$-statistic is larger than the $t$-distribution. 

DRIVER'S ELECTROMYOGRAM AND MOVEMENT TRAJECTORY

The results of $t$-test, as well as the Pearson correlation coefficient $R$ of each index are shown in Table II. It can be seen that all of the five indices show statistical linear relationship with the SCR at 0.05 level $\left(\mathrm{p}\left(\mathrm{H}_{0}\right)<0.05\right)$.

For EMG indices, there is a positive correlation between the average steering efficiency and the SCR, which means that when the steering efficiency is higher, the driver may have a better steering comfort. On the contrary, the average muscle co-contraction has a negative correlation with SCR, which means that when muscle co-contraction increases, the driver may actually get a worse steering comfort. In fact, while the steering system fails to respond to the driver's maneuver and the driver's steering comfort is lowered significantly, the driver may tend to increase the stiffness of his upper limb through a higher level of muscle antagonism to improve his control ability. During this process, the driver's muscle co-contraction becomes more intense, and his steering comfort may become worse.

For movement indices, all of the three have a negative correlation with SCR, which means that when the average velocity (or the average acceleration, average jerk) increases, the driver's steering comfort feeling may become worse. This indicates that the driver may prefer a slower, smoother steering process, instead of intense operations.

However, for all of the five indices, the linear relationship is very weak as the Pearson's $R$ is very small (less than 0.3), which indicates that each single index cannot be effectively applied to evaluation of steering comfort. This may be because the driver tends to adopt some complicated control strategies during the steering maneuver, instead of simpler strategies to improve one particular index. In addition, according to Fig. 10 it can be seen that the standard deviations of the indices are very large even for the same SCR, which makes it difficult to develop an accurate objective evaluation approach based on one single index.

Therefore, although it has been found that there is a significant correlation between objective indices and subjective evaluations, it is still necessary to build some models for the objective evaluation of steering comfort. In Section V, a preliminary research on the ANN model of steering comfort is conducted.

TABLE II

SIGNIFICANCE OF RELATIONSHIP BETWEEN EVALUATION INDICES AND STEERING COMFORT RATINGS

\begin{tabular}{lll}
\hline \hline Evaluation index & Significance & Pearson $R$ \\
\hline Steering Efficiency & 0.005 & 0.230 \\
Muscle Co-contraction & 0.000 & -0.283 \\
Average Velocity & 0.023 & -0.189 \\
Average Acceleration & 0.013 & -0.207 \\
Average Jerk & 0.007 & -0.224 \\
\hline \hline
\end{tabular}


Fig. 10 The trend of the EMG indices and the movement indices under different SCRs. Note that in the current experiment, there are only two samples rated 4 , and there are no samples rated 1,9 , and 10 .

\section{OBjective Evaluation Using ANN}

Considering that there may be complex, nonlinear relationships between quantitative evaluation indices and the SCR, a nonlinear model may be able to predict the subjective SCR. In this paper, an ANN model is established because of its good learning ability for the complex nonlinear relationships.

\section{A. Topology of ANN evaluation model}

The primary consideration of neural network design is to determine the topology type of the network. A common classification of the ANNs topology is based on the interconnected method of their elements [33]. There are mainly the following types of ANNs: multilayer perceptron (MLP), Hopfield, Kohonen, etc., of which MLP has been proved more effective in the prediction of automotive seat comfort [22]. The MLP is adopted as the topology of the prediction model, which consists of one input layer, several hidden layers, and one output layer.

Then, the number of the hidden layers and the neurons should be determined. Generally, there is no definite conclusion on the relationship between neuron numbers and prediction accuracy. Too few neurons may limit the learning ability, while too many neurons may lead to data over-fitting and hinder the generalization. Furthermore, once the network reaches a certain sufficient scale, the prediction accuracy can no longer be significantly improved by increasing the neurons [37]. After trying many times, the topology of ANN model is selected as one hidden layer with 30 neurons.

\section{B. Training, validation and test}

Levenberg-Marquardt (LM) algorithm, a second-order 
gradient method, is used for the network training. The input matrix of the network consists of all of the five evaluation indices, and the SCR is set as the training target.

The samples collected from 21 subjects are randomly divided into three groups by the ratio of $70 \%, 15 \%$, and $15 \%$ :

1) Training group (70\%), which is loaded into the network during the model training, and the weight of each connection between neurons is adjusted according to its error.

2) Validation group (15\%), which is used to measure the generalization ability of network and halt the training process when its generalization stops improving.

3) Test group (15\%), which have no effect on the training and therefore they can provide independent measure of the network performance during and after the training process.

In addition, in order to verify the model performance for the drivers whose data are not included in model training, both the validation group and the test group are further divided into two subgroups. The subgroups include: 1) subgroup (a), which consists of the randomly selected samples from 19 subjects; 2) subgroup (b), in which the samples are from the other two subjects (one subject for validation, and one for test) whose samples are not included in the model training, but only used in the validation or the test.

\section{Feasibility of the ANN model}

Based on the samples of the steering experiment, an ANN model is built, and its performance is shown in Fig. 11 and in Table III. The performance is assessed by the mean square error (MSE) as well as the coefficient of determination $R^{2}$. It can be seen that the prediction of SCR (the output of the ANN) shows a strong correlation with the subjective $\mathrm{SCR}$, while $R^{2}$ is 0.593 and MSE is 0.627 for all sample groups. For the training group and all sample groups, $R^{2}$ and MSE are almost the same. However, for the validation group and the test group, the ANN's performance is somewhat different.

In the test group, the model performance is acceptable for the subjects whose samples are included in the model training (in the group of test (a), $R^{2}$ and MSE are acceptable). On the other hand, for the subject not included in model training, the performance shows some decline (in the group of test (b), $R^{2}$ is too small. Such decline indicates that the current ANN approach is very dependent on the database. When the sample information is not included in the database, its performance might decline significantly.

In the validation group, the performance is good for validation (b), but for validation (a) it is not satisfactory. This indicates that the model's generalization is still in need of improvements.

In summary, the predicting performance of the ANN model is acceptable, and the ANN model may prove to be a potential candidate for a quantitative, objective evaluation approach to evaluating the driver steering comfort. However, since its predicting accuracy is not sufficient and the model generalization is insufficient, the current approach needs further improvement (such as increasing the number of subjects to form a larger database to cover more information of different drivers and different driving conditions, establishing other efficient evaluation indices, or adjusting the topology of ANN, etc.).
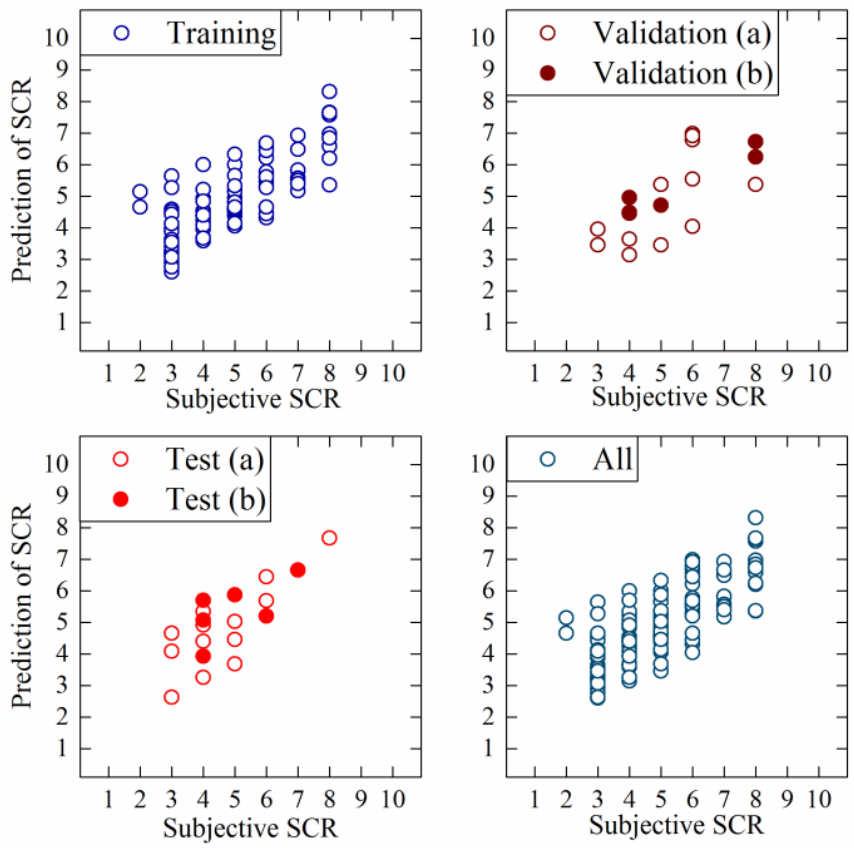

Fig. 11 Verification of the performance of ANN model on predicting the SCR. In the group of "Validation" and "Test", subgroup "(a)" consists of the random selected samples from 19 subjects; and in subgroup "(b)", the samples are from the subject whose data is independent of the model training.

TABLE III

PERFORMANCE OF THE ANN MODEL ON PREDICTING THE STEERING COMFORT RATINGS

\begin{tabular}{llll}
\hline \hline Sample group & Sample number & $R^{2}$ & MSE \\
\hline Training & 88 & 0.624 & 0.576 \\
Validation (a) & 13 & 0.478 & 1.256 \\
Validation (b) & 6 & 0.633 & 0.454 \\
Validation (a\&b) & 19 & 0.482 & 0.964 \\
Test (a) & 13 & 0.638 & 0.699 \\
Test (b) & 6 & 0.433 & 0.597 \\
Test (a\&b) & 19 & 0.593 & 0.647 \\
\hline Overall & 126 & 0.593 & 0.627 \\
\hline \hline
\end{tabular}

\section{CONCLUSIONS}

This paper investigates the relationships between the driver's subjective steering comfort and the driver's physiological characteristics. An ANN model is built based on quantitative indices, and it proves a promising candidate for establishing an objective evaluation method of steering comfort.

First, based on measured EMG and movement trajectories of driver's upper limbs, five evaluation indices are established for steering comfort, and the linear regression analysis is conducted for each evaluation index. The results show that all the indices are in statistical linear relationship with the SCR at the 0.05 level. There is a positive correlation between steering efficiency and SCR, whereas the remaining indices are 

DRIVER'S ELECTROMYOGRAM AND MOVEMENT TRAJECTORY

negatively correlated with SCR. This indicates that these quantitative indices are good descriptions of driver's physiological characteristics and may be useful in evaluating steering comfort.

However, due to the weak Pearson's $R$ and the large standard deviations, the indices cannot be used directly in the objective evaluation. The reason might be that the driver's maneuvers may involve very complicated control strategies during steering, instead of acting to achieve an individual aim, such as increasing the smoothness of movement. Therefore, an ANN model is built to find a better approach of objective evaluation, since the ANN performs well on learning nonlinear relationship among variables. The results show that the ANN model has acceptable performance in predicting the SCR, and it may prove to be a feasible way to evaluate steering comfort.

According to the correlation between quantitative indices and SCR, as well as acceptable predicting performance of the ANN model, we can conclude that it is possible to establish an objective evaluation approach for vehicle steering comfort. However, the current ANN approach is data-based, and it might only be effective for a specific driving condition and for a specific subject set. Further investigation should be made to establish a general, objective evaluation method. In our future work, experiments on real vehicles will be conducted with broader driving conditions to achieve more generic evaluation methods.

\section{ACKNOWLEDGMENT}

The authors would like to thank the National Natural Science Foundation of China (51375009) and Tsinghua University Initiative Scientific Research Program (2014z21039), and thank the support of JTEKT Co., Ltd, Japan.

\section{REFERENCES}

[1] M. Lu, K. Wevers, and R. V. D. Heijden, "Technical Feasibility of Advanced Driver Assistance Systems (ADAS) for Road Traffic Safety," Transport. Plan. Techn., vol. 28, no. 3, pp. 167-187, Jun. 2005.

[2] V. A. Butakov and P. Ioannou, "Personalized Driver/Vehicle Lane Change Models for ADAS," IEEE Trans. Veh. Technol., vol. 64, no. 10, pp. 4422-4431, Oct. 2015.

[3] Y. Liu, T. Li, Y. Yang, X. Ji, and J. Wu., " Estimation of tire-road friction coefficient based on combined APF-IEKF and iteration algorithm," Mech. Syst. Signal Process., vol. 88, no. 3,pp. 25-35, May 2017.

[4] R. Kuiper, D. Heck, I. Kuling, and D. Abbink, "Evaluation of Haptic and Visual Cues for Repulsive or Attractive Guidance in Nonholonomic Steering Tasks," IEEE Trans. Human-Mach. Syst., vol. 46, no. 5, pp.672-683, Oct. 2016.

[5] D. A. Abbink, D. Cleij, M. Mulder, and M. M. van Paassen, "The importance of including knowledge of neuromuscular behaviour in haptic shared control," in Proc. IEEE Int. Conf. System, Man, Cybern., 2012, pp. 3350-3355.

[6] M. Alirezaei, M. Corno, D. Katzourakis, A. Ghaffari, and R. Kazemi, "A robust steering assistance system for road departure avoidance," IEEE Trans. Veh. Technol., vol. 61, no. 5, pp. 1953-1960, Jun. 2012.

[7] D. Katzourakis, J. de Winter, M. Alirezaei, M. Corno, and R. Happee,"Road-departure prevention in an emergency obstacle avoidance situation," IEEE Trans. Syst., Man, Cybern., Syst., vol. 44, no. 5, pp. 621629, May 2014.

[8] B. A. Forsyth and K. E. Maclean, "Predictive haptic guidance: intelligent user assistance for the control of dynamic tasks," IEEE Trans. Vis. Comput. Graphics, vol. 12, no. 1, pp. 103-113, 2006.
[9] S. Schnelle, J. Wang, H. Su, and R. Jagacinski, "A Personalizable Driver Steering Model Capable of Predicting Driver Behaviors in Vehicle Collision Avoidance Maneuvers," IEEE Trans. Human-Mach. Syst., to be published.

[10] S. M. Petermeijer, D. A. Abbink, M. Mulder, and J. C. F. de Winter, "The effect of haptic support systems on driver performance and behavior: A literature review," IEEE Trans. Haptics, vol. 8, no. 4, pp. 467-479, 2015.

[11] F. Mars, M. Deroo, and J. M. Hoc, "Analysis of Human-Machine Cooperation When Driving with Different Degrees of Haptic Shared Control," IEEE Trans. Haptics, vol. 7, no. 3, pp. 324-333, 2014.

[12] D. J. Cole, "A path-following driver-vehicle model with neuromuscular dynamics, including measured and simulated responses to a step in steering angle overlay," Veh. Syst. Dyn., vol. 50, no. 4, pp. 573-596, Apr. 2012.

[13] N. Kim and D. J. Cole, "A model of driver steering control incorporating the driver's sensing of steering torque," Veh. Syst. Dyn., vol. 49, no. 10, pp. 1575-1596, Oct. 2011.

[14] X. Na and D. J. Cole, "Game-Theoretic Modeling of the Steering Interaction Between a Human Driver and a Vehicle Collision Avoidance Controller," IEEE Trans. Human-Mach. Syst., vol. 45, no. 1, pp. 25-38, Feb. 2015.

[15] M. Kolich, N. Seal, and S. Taboun, "Automobile seat comfort prediction: statistical model vs. artificial neural network," Appl. Ergon., vol. 35, no. 3, pp. 275-284, 2004.

[16] J. M. Porter and D. E. Gyi, "Exploring the optimum posture for driver comfort," Int. J. Vehicle Design, vol. 19, no. 3, pp. 255-266, 1998.

[17] S. J. Park, C. Kim, C. J. Kim, and J. W. Lee, "Comfortable driving postures for Koreans," Int. J. Ind. Ergonom., vol. 26, no. 4, pp. 489-497, 2000.

[18] D. Mohamad, B. M. Deros, and D. A. Wahab, "Integration of Comfort into a Driverâs Car Seat Design Using Image Analysis," Am. J. Applied Sci., vol. 7, no. 7, pp. 937-942, 2010.

[19] D. Mohamad, B. M. Deros, D. D. I. Daruis, N. F. Ramli, and E. H. Sukadarin, "Comfortable Driver's Car Seat Dimensions Based on Malaysian Anthropometrics Data," Iran J. Public Health, vol. 45, suppl. issue no. 1, pp. 106-113, Feb. 2016.

[20] S. Schmidt, M. Amereller, M. Franz, R. Kaiser, and A. Schwirtz, "A literature review on optimum and preferred joint angles in automotive sitting posture," Appl. Ergon., vol. 45, no. 2, pp. 247-260, 2014.

[21] G. Kyung, M. A. Nussbaum, and K. Babski-Reeves, "Driver sitting comfort and discomfort (part I): Use of subjective ratings in discriminating car seats and correspondence among ratings," Int. J. Ind. Ergonom., vol. 38, no. 5, pp.516-525, 2008.

[22] M. Kolich, "Predicting automobile seat comfort using a neural network," Int. J. Ind. Ergonom., vol. 33, no. 4, pp. 285-293, 2004.

[23] G. Kyung and M. A. Nussbaum, "Specifying comfortable driving postures for ergonomic design and evaluation of the driver workspace using digital human models," Ergonomics, vol. 52, no. 8, pp. 939-953, Aug. 2009.

[24] G. Kyung and M. A. Nussbaum, "Driver sitting comfort and discomfort (part II): Relationships with and prediction from interface pressure," Int. J. Ind. Ergonom., vol. 38, no. 5, pp. 526-538, 2008.

[25] S. J. Park, S. N. Min, M. S. Subramaniyam, H. Lee, et al., "Driving Posture Measurement using 3D Scanning Measuring Technique," $S A E$ Int. J. Passeng. Cars - Mech. Syst., vol. 8, no. 2, Jul. 2015.

[26] N. Alessandro and M. Sandro, "Postural Comfort Inside a Car: Development of an Innovative Model to Evaluate the Discomfort Level," SAE Int. J. Passeng. Cars - Mech. Syst., vol. 2, no. 1, pp. 1065-1070, 2009.

[27] Y. Yang, Y. Liu, M. Wang, R. Ji, and X. Ji, "Objective Evaluation Method of Steering Comfort Based on Movement Quality Evaluation of Driver Steering Maneuver," Chin. J. Mech. Eng., vol. 27, no. 5, pp. 1027-1037, 2014.

[28] Y. Liu, X. Ji, R. Hayama, T. Mizuno, and L. Lou, "Function of shoulder muscles of driver in vehicle steering maneuver," Sci. China Tech Sci., vol. 55, no. 12, pp. 3445-3454, Dec. 2012.

[29] Y. Liu, X. Ji, R. Hayama, and T. Mizuno, "A Novel Estimating Method for Steering Efficiency of the Driver with Electromyography Signals," Chin. J. Mech. Eng., vol. 27, no. 3, pp. 460-467, 2014.

[30] Y. Liu, X. Ji, R. Hayama, T. Mizuno, and S. Nakano, "Method for measuring a driver's steering efficiency using electromyography," Proc. IMechE, Part D: J. Automobile Engineering, vol. 228, no. 10, pp. 1170-1184, 2014.

[31] M. Franz, R. Zenk, P. Vink, and S. Hallbeck, "The Effect of a Lightweight Massage System in a Car Seat on Comfort and 
Electromyogram," J. Manipulative Physiol. Ther., vol. 34, no. 2, pp. 107-113, 2011.

[32] A. J. Pick, and D. J. Cole, "Measurement of Driver Steering Torque Using Electromyography," ASME J. Dyn. Syst., Meas., Control, vol. 128, no. 4, pp. 960-968, Dec. 2006.

[33] M. Meireles, P. Almeida, and M. G. Simões, "A Comprehensive Review for Industrial Applicability of Artificial Neural Networks," IEEE Trans. Ind. Electron., vol. 50, no. 3, pp. 585-601, Jun. 2003.

[34] L. Li, Z. Zhu, X. Wang, et al., "Identification of a driver's starting intention based on an artificial neural network for vehicles equipped with an automated manual transmission," Proc. IMechE, Part D: J. Automobile Engineering, vol. 230, no. 10, pp. 1417-1429, 2015.

[35] L. Li, Z. Zhu, Y. Chen, et al., "Engagement control of automated clutch for vehicle launching considering the instantaneous changes of driver's intention," ASME J. Dyn. Syst., Meas., Control, vol. 139, no. 2, pp. 1-12, Feb. 2017.

[36] A. J. Pick and D. J. Cole, "Dynamic properties of a driver's arms holding a steering wheel," Proc. IMechE, Part D: J. Automobile Engineering, vol. 221, no. 12, pp. 1475-1486, 2007.

[37] S. J. Rutherford and D. J. Cole, "Modelling nonlinear vehicle dynamics with neural networks," Int. J. Vehicle Design, vol. 53, no. 4, pp. 260-287, 2010.

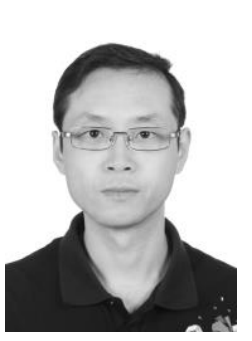

Yahui Liu received his B.S. degree from Jilin University, China, in 2003 and Ph.D. degree from Beihang University, China, in 2009 respectively. He is currently an Assistant Professor at State Key Laboratory of Automotive Safety and Energy, Tsinghua University, China.

His research interests include driver-vehicle system dynamics, steering system, and actuator design of ADAS.



Qi Liu received his B.S. degree in automotive engineering from Tsinghua University, China, in 2016. He is currently working toward the M.S. degree in mechanical engineering at State Key Laboratory of Automotive Safety and Energy, Tsinghua University, China.

His research interests include drivervehicle system and automated driving.

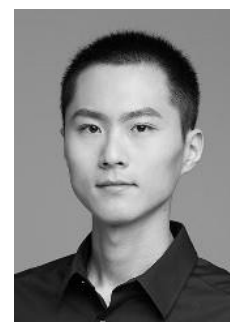

Chen Lv is currently a research fellow at Advanced Vehicle Engineering Center, Cranfield University, UK. He received the $\mathrm{Ph} . \mathrm{D}$. degree at Department of Automotive Engineering, Tsinghua University, China, in 2016. From 2014 to 2015, he was a visiting researcher at EECS Dept., University of California, Berkeley.

His research focuses on electrified vehicles and automated driving.

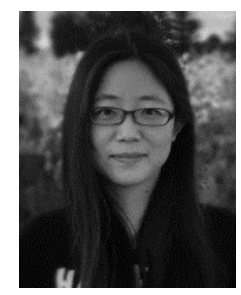

Minghui Zheng is currently a Ph.D. Candidate in the Department of Mechanical Engineering at University of California, Berkeley. She received her B.S. degree in Engineering Mechanics and M.S. degree in Control Science and Engineering from Beijing University of Aeronautics and Astronautics, Beijing, China.

Her research interests include advanced learning and control with applications to high-precision systems and robotics.

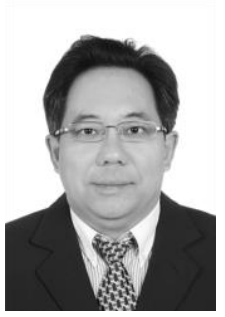

Xuewu Ji received his Ph.D. degree in automotive engineering from Jilin University, China, in 1994. He is currently an Associate Professor at State Key Laboratory of Automotive Safety and Energy, Tsinghua University, China.

His research interests include steering system and vehicle system dynamics. 
2017-10-05

\title{
A study on objective evaluation of vehicle steering comfort based on
} driver's electromyogram and movement trajectory

\author{
Liu, Yahui
}

IEEE

Liu Y, Liu Q, Lv C, Zheng M, Ji X, A study on objective evaluation of vehicle steering comfort based on driver's electromyogram and movement trajectory, IEEE Transactions on

Human-Machine Systems, Vol. 48, Issue 1, February 2018, pp. 41-49

http://dx.doi.org/10.1109/THMS.2017.2755469

Downloaded from Cranfield Library Services E-Repository 